Este artículo presenta los resultados de un estudio cualitativo de carácter ARTICULOS · 163-181

exploratorio cuyo objetivo era obtener información sobre el perfil del traductor médico y las competencias necesarias para el ejercicio profesional. Para ello, se ha entrevistado a doce traductores de diversos perfiles formativos (médicos, biólogos y traductores) que trabajan como autónomos o en plantilla para organismos diversos. El estudio nos ha permitido obtener datos variados sobre el ejercicio de la traducción médica (géneros y temas traducidos, dificultades encontradas, requisitos exigidos, clientes, etc.) y ha puesto de manifiesto interesantes diferencias en el desarrollo profesional de los traductores según el perfil formativo o el régimen laboral. Los resultados muestran, además, que la adquisición de conocimientos médicos básicos o el dominio de las fuentes de documentación son algunas de las competencias que los informantes consideran clave para el ejercicio profesional.

PALABRAS CLAVE: perfil del traductor médico profesional, competencias, estudio cualitativo, formación en traducción médica.

\title{
El perfil y las competencias del traductor médico desde el punto de vista de los profesionales: una aproximación cualitativa ${ }^{*}$
}

The profile and competences of medical translators from the professionals' point of view: A qualitative approach

Ana Muñoz-MiQueL

Universitat Jaume I

This paper presents the results of an exploratory qualitative study aimed to gather information on the medical translator profile and the competences required for the practice of the profession. To do so, twelve translators with different training profiles (physicians, biologists and translators) working as freelancers or staff workers in different organizations were interviewed. The study allowed us to obtain data about the practice of medical translation (genres and topics translated, difficulties encountered, employers' requirements, clients, etc.), and yielded some interesting differences in the trans/ators' professional career depending on the training profile or the employment status. The results also show that acquiring background medical knowledge or mastering medical documentary sources are key competences for professional practice, according to the respondents' opinions.

KEY WORDS: Professional medical translator profile, competences, qualitative study, medical translation training. \footnotetext{
34200), financiado por el Ministerio de Economía y Competitividad.

Este estudio ha sido parcialmen financiado por la Universitat Jaume I (PREDOC/2009/43) y se ha realizado en el marco de proyecto Análisis de necesidades y propuesta de recursos de información escrita para pacientes en el ámbito de la Oncología (FFI2or2-
} 


\section{INTRODUCCIÓN}

Hoy en día, el nuevo escenario de educación universitaria determina que la formación se articule en torno a la adquisición de competencias y en función de perfiles profesionales existentes en el mercado. Por esta razón, cada vez se hace más necesario obtener datos de los profesionales en ejercicio que contribuyan a planificar la formación de manera más coherente con lo que sucede en el mundo laboral. En traducción médica, sin embargo, apenas se han realizado investigaciones de este tipo, y los trabajos existentes se centran en describir las competencias o el perfil que debería tener el traductor médico basándose en la intuición o la experiencia personal, pero no en datos empíricos que puedan corroborar de manera más objetiva cualquier valoración.

Ante este vacío investigador y partiendo de la premisa de que obtener información por parte de los profesionales en ejercicio puede contribuir a que se tomen decisiones más informadas sobre las competencias necesarias para ejercer, en este artículo presentamos los resultados de un estudio cualitativo exploratorio cuyo objetivo era obtener datos sobre el perfil y las competencias del traductor médico mediante la realización de entrevistas a una muestra de traductores profesionales. El artículo se estructura de la siguiente manera: tras una breve introducción a la traducción médica desde un punto de vista formativo y profesional (apartado 2), se describe la metodología utilizada y las características de los informantes (apartado 3). A continuación, se presentan los datos obtenidos en las entrevistas agrupados en dos grandes bloques: perfil y desarrollo profesional, por un lado, y competencias destacadas, por otro (apartado 4). Por último, se interpretan los principales hallazgos y se presentan las conclusiones más relevantes del estudio (apartado 5).

\section{ASPECTOS FORMATIVOS Y PROFESIO- NALES DE LA TRADUCCIÓN MÉDICA}

La traducción médica es, sin duda, una actividad fundamental para el desarrollo de la comunicación y el conocimiento médicos en todas sus vertientes (Montalt, 2007: 213): desde la investigación clínica y el desarrollo de medicamentos hasta la educación del paciente, la promoción de la salud o la atención sanitaria. A juzgar por los resultados de estudios de mercado como el realizado por la Agrupación de Centros Especializados en Traducción (Orf, 2005), se trata de una actividad en constante crecimiento en la que la industria farmacéutica ocupa un lugar destacado. El sector editorial también se revela como uno de los más fructíferos, pues, según el Instituto Nacional de Estadística, en 2012 el número de libros de ciencias médicas y sanidad traducidos del inglés superaba notablemente al de otros ámbitos científico-técnicos. En organismos internacionales como la Organización Mundial de la Salud (oms) o la Organización Panamericana de la Salud (OPs) también traducen anualmente un generoso volumen de documentos. En la OPS, por ejemplo, la media de palabras anual ronda los cinco millones (Lozano, 2009: 88), una cifra que va en aumento según los datos publicados en el sitio web de la organización. ${ }^{\mathrm{I}}$ Asimismo, otros sectores, como los centros de investigación, los hospitales, las instituciones europeas, las empresas de investigación clínica (CRO) o las universidades, generan a diario gran cantidad de información médico-sanitaria que necesita traducirse por razones diversas. La traducción médica se presenta, pues, como una de las especialidades más fructíferas en lo que a posibilidades de trabajo y de desarrollo profesional se refiere.

\footnotetext{
I Véase: http://new.paho.org/blogs/
}

$\mathrm{kmc} /$ ? $\mathrm{p}=396 \&$ lang=es. [Fecha de la última consulta: $30 \mathrm{de}$ noviembre de 20I3] 
Desde un punto de vista disciplinario y dado el parentesco indudable que guarda con la traducción científico-técnica, la traducción médica ha quedado tradicionalmente diluida como una subespecialidad dentro de aquélla (Gamero, 20or; Mayoral y Diaz, 2orr). Sin embargo, cada vez son más los académicos y profesionales del ámbito que inciden en resaltar las "características que le son propias» (Montalt, 2009: 172) y conferir a la traducción médica el estatus de especialidad acotada, con entidad propia y con necesidades formativas particulares. Así, los autores aluden a su complejidad, "propia de los fundamentos científicos de la Medicina» (Alarcón, I998: I033), su amplitud, la especificidad de las nociones y la terminología médicas, la existencia de géneros textuales prototípicos del ámbito o su amplia demanda en el mercado, para resaltar la necesidad de contar con profesionales altamente cualificados y específicamente formados en esta especialidad (Pilegaard, 2000; Lee-Jahnke, 200I; Navarro, 2002; Mayor, 2003; Montalt y González, 2007; Albarrán, 2012). Como afirma Franco (2010: 157):

La TeI médica constituye una de las áreas profesionales más importantes, tanto cuantitativamente, por la cantidad de trabajo potencial que aglutina, como cualitativamente, por la complejidad de traducir o interpretar en un campo que exige conocimientos especializados que no están al alcance de cualquier persona por el simple hecho de ser culta, como sí puede suceder en otras áreas de la TeI.

Tradicionalmente, han sido los profesionales de la medicina con conocimientos de idiomas los que se han encargado de satisfacer las necesidades de traducción médico-sanitaria, hecho que se ha debido, en gran parte, a la juventud de los estudios de traducción y a la escasa oferta de formación especializada. Afortunadamente, en los últimos años la situación ha empezado a cambiar gracias a la inclusión progresiva de la traducción médica en la formación universitaria de posgrado. Prueba de ello, en el caso de España, es la existencia de posgrados específicos, como el Máster Oficial en Traducción Médico-Sanitaria de la Universitat Jaume $\mathrm{I}^{2} \mathrm{o}$ el Programa Propio de Posgrado de Traducción Biomédica y Ciencias de la Salud que organiza el idec de la Universitat Pompeu Fabra. ${ }^{3}$ Asimismo, cada vez son más los másteres que poco a poco le van cediendo espacio a la traducción médica mediante la inclusión en los planes de estudio de itinerarios o de materias de especialización en esta rama. ${ }^{4}$

\section{I. La vinculación entre profesión y formación: una asignatura pendiente}

Como apuntábamos en la introducción, en el contexto de educación superior en el que se enmarcan actualmente los posgrados mencionados, la determinación de perfiles profesionales y la formación por competencias han adquirido un peso fundamental a la hora de diseñar el currículo de una manera más acorde con lo que sucede en la realidad profesional. La determinación del perfil profesional, como afirman Yániz y Villardón (2006: 20): «ayuda a identificar los conocimientos, habilidades y

\footnotetext{
2 http://www.tradmed.uji.es/. [Fecha de la última consulta: 30 de noviembre de 2013]

$3 \mathrm{http} / /$ www.idec.upf.edu/programa-de-postgradode-traduccion-biomedica-y-ciencias-de-la-salud. [Fecha de la última consulta: 30 de noviembre de 20I3]

4 Como el Máster Universitario en Traducción Profesional e Institucional de la Universidad de Valladolid, el Máster en Comunicación Intercultural, Interpretación y Traducción en los Servicios Públicos de la Universidad de La Laguna, el Máster en Traducción para el mundo editorial de la Universidad de Málaga o el Máster en Comunicación Intercultural, Interpretación y Traducción en los sspe de la Universidad de Alcalá de Henares.
} 
actitudes que componen las competencias o capacitación necesaria para desarrollarlo». Y, para ello, obtener información de los profesionales en ejercicio resulta esencial, en tanto que, como señalan estas autoras (2006: I8-I9):

- las actividades profesionales pueden ser descritas en términos de funciones y tareas, $\mathrm{y}$ requieren una serie de conocimientos, habilidades y actitudes, identificables de manera concreta, que el profesional debe poseer;

- los expertos, es decir, aquellos que han desarrollado la profesión durante un tiempo determinado y de manera efectiva, son la mejor fuente para describir un perfil profesional y, por ende, una profesión.

El establecimiento de un mayor diálogo entre el mundo académico y el profesional es, pues, un elemento clave de la reforma universitaria, y en este diálogo son las competencias las que se convierten en ese «discurso que sirve de nexo" (Calvo, 2010: 149). Según esta autora, la conexión entre perfiles profesionales y competencias viene determinada por una «concepción cíclica» (2010: I50) de la relación entre sociedad/mercado de trabajo y formación, por la cual, tras un análisis del mercado laboral, se identificarían los perfiles profesionales requeridos en el mercado. Estos posteriormente se traducirían en una serie de competencias susceptibles de ser adquiridas por los estudiantes. Así pues, la realización de estudios que persigan obtener información sobre el mercado de la traducción, las competencias que requieren los empleadores o los perfiles profesionales más demandados, adquieren más importancia, si cabe, en este nuevo contexto. Buena muestra de ello son el proyecto Tuning (González y Wagenaar, 2003) o el Libro Blanco del título de Grado en Traducción e Interpretación (Muñoz Raya, 2004), en los que se establecen una serie de perfiles profesionales ${ }^{5} \mathrm{y}$ se determinan las competencias genéricas y específicas más valoradas a partir de los resultados de encuestas realizadas a empleadores, profesores y licenciados. Asimismo, iniciativas más recientes como el proyecto Optimale (Optimising professional translator training in a multilingual Europe), ${ }^{6}$ en el que se esbozan las competencias demandadas en el mercado a partir de un estudio cuantitativo, evidencian los esfuerzos por trazar puentes entre el ámbito académico y el profesional. Todas estas propuestas, sin embargo, se centran en un perfil transversal a las distintas especialidades de traducción y, por tanto, podrían no reflejar las especificidades de la traducción médica ni establecer una jerarquización de las competencias en función de las prioridades y necesidades que, según autores como Navarro y Hernández (1997), Lee-Jahnke (1998), Montalt y González (2007) o Rouleau (20I2), plantea esta especialidad.

Los estudios centrados en las competencias o el perfil profesional del traductor médico son más bien escasos. Lo que abundan son trabajos en los que las competencias se ofrecen como paso previo al análisis de otras cuestiones (Muñoz, Montalt y García Izquierdo, 20I2: I08), por lo que estas no constituyen el eje fundamental de ninguna investigación. Asimismo, en la mayoría de casos dichas competencias surgen de una dicotomía que ha cosechado intensos debates en la bibliografía académica (Fischbach, I998: 4): qué profesional se ajusta mejor al ejercicio de esta actividad, si el formado en medicina o ciencias afines con

5 Los perfiles profesionales a los que, a priori, la formación en Traducción e Interpretación debería dar respuesta se determinan únicamente en el Libro Blanco.

6 Proyecto vinculado a la creación del Máster Europeo de Traducción (EMT), un marco de referencia para los másteres en traducción en el que se establecen las competencias que deberían adquirirse en los programas de posgrado. 
conocimientos lingüísticos o el formado en traducción o filología con conocimientos médicos o científicos. En estas contribuciones, ${ }^{7}$ como ya hemos apuntado, los autores suelen describir las características que debería tener el traductor médico fundamentándose principalmente en la intuición o en su propia experiencia como profesionales, docentes o académicos. Se trata, por tanto, de opiniones subjetivas que, pese a su indudable interés, no se complementan con datos empíricos que reflejen más objetivamente las cualidades observadas en los traductores profesionales (Mayoral, 20or: III). En lo que atañe a los perfiles y salvo algunas excepciones (como el trabajo de O'Neill de I998), tampoco existen estudios que aporten datos sistemáticos sobre las tareas que desempeñan los traductores médicos en el ejercicio profesional y que contribuyan, de esa manera, a determinar su perfil.

Como vemos, en el ámbito de la traducción médica, tender puentes entre la realidad profesional y el contexto académico es, todavía, una asignatura pendiente que este trabajo pretende contribuir a superar mediante la investigación cualitativa. A continuación, explicaremos con detalles la metodología utilizada en el estudio.

\section{METODOLOGÍA}

El objetivo de los métodos cualitativos es comprender, describir y descubrir los aspectos subjetivos más significativos de una situación particular, y determinar qué piensan los participantes de dicha situación. Para ello, se suele recopilar una amplia cantidad de datos y se efectúan análisis inductivos, en tanto que se procede de lo más específico a lo más general (Creswell, 2007: 43).

\footnotetext{
7 Para una revisión de algunas de las contribuciones más significativas, véase: Muñoz Miquel (2009) o Muñoz, Montalt y García Izquierdo (20I2).
}

Entre los distintos instrumentos para obtener datos cualitativos (entrevistas, encuestas, grupos de discusión, observaciones, análisis de contenido, etc.), se ha escogido la entrevista semiestructurada. Este tipo de entrevista permite definir previamente las preguntas en un guión, pero la secuencia y formulación de estas puede variar en función de cada informante. Así, el investigador tiene la flexibilidad suficiente para poder profundizar en cuestiones relevantes mediante la formulación de nuevas preguntas.

\section{I. Criterios de selección de los informantes}

Para seleccionar a los informantes se utilizó la estrategia de máxima variación (Creswell, 2007: I26) como técnica de muestreo, en la que se determinan criterios diversos, esenciales para la investigación, y se selecciona un cierto número de participantes que los cumplan, a fin de responder a la heterogeneidad de la población. Los criterios que debían cumplir los traductores seleccionados fueron los siguientes:

- que se dedicaran profesionalmente a la traducción médica;

- que representaran los perfiles formativos más comunes: traductores formados en lenguas o traducción y traductores formados en medicina o ciencias afines;

- que representaran diversos perfiles profesionales (autónomos y de plantilla), y

- que trabajaran con la combinación lingüística inglés-español (lo cual no excluía otras combinaciones).

En función de estos criterios, se seleccionó a doce informantes (siete mujeres y cinco hombres) ${ }^{8}$ residentes en países diversos (Argen-

\footnotetext{
8 Durante la exposición de los resultados (apartado 4) y para garantizar el anonimato de los informantes, se hará referencia a todos ellos utilizando el masculino gramatical genérico.
} 
TABLA I. PRINCIPALES CARACTERÍSTICAS DE LOS INFORMANTES.

$\begin{array}{ll}\mathrm{TFCI}_{1} & \text { Licenciado en Medicina. } \\ \mathrm{TFC}_{2} & \text { Licenciado en Medicina. } \\ \mathrm{TFC}_{3} & \text { Licenciado en Medicina. } \\ \mathrm{TFC}_{4} & \text { Licenciado en Medicina. } \\ & \\ \mathrm{TFC}_{5} & \text { Licenciado en Biología. } \\ & \text { Doctor en Biología Molecular. }\end{array}$

TFLI Licenciado en Traducción e Interpretación.

TFL2 Licenciado en Traducción e Interpretación. Doctor en Traducción.

TFL3 Licenciado en Traducción.

TFL4 Licenciado en Traducción e Interpretación.

TFL5 Licenciado en Traducción e Interpretación.

TFL6 Licenciado en Traducción e Interpretación. Máster en Interpretación de conferencias. TFL7 Licenciado en Traducción e Interpretación.
Autónomo.

$>30$

Autónomo.

23

En plantilla para una editorial médica.

I3

En plantilla para el servicio de traducción de una institución europea.

En plantilla para un laboratorio farmacéutico.

Autónomo. I5

En plantilla para un organismo internacional.

En plantilla para un organismo internacional de salud pública.

Autónomo.

Autónomo. Socio de una empresa IO de traducción médica.

Autónomo. Socio de una empresa Io de traducción médica.

Autónomo. Socio de una empresa de traducción médica.

* Nos referimos a los años de ejercicio regular e ininterrumpido como traductores de textos médico-sanitarios. Muchos de los informantes tenían más años de experiencia previa traduciendo otras especialidades, que no hemos considerado para el cómputo.

tina, España, Estados Unidos, Luxemburgo, Reino Unido y Suiza). Siete eran Licenciados en Traducción e Interpretación y cinco habían recibido formación científica, y entre todos había autónomos, socios de empresas de traducción médica y traductores de plantilla. La tabla I resume las características principales de los informantes, que se han agrupado según su perfil formativo (TFC identifica a los traductores de formación científica y TFL, a los de formación lingüística):

Como puede observarse, prácticamente todos los informantes eran expertos, ya que, como señalaron durante las entrevistas, acumulaban un mínimo de diez años de ejercicio regular y continuado (Bloom, I985) en el ámbi- to de la traducción médica. Consideramos, por tanto, que sus respuestas (véase apartado 4) están sólidamente fundamentadas en su amplia experiencia como traductores médicos profesionales.

\subsection{Procedimiento}

Las entrevistas se realizaron entre los meses de octubre de 2011 y junio de 20I2. Los posibles informantes fueron seleccionados a partir de la observación de un foro especializado en traducción médica. Todos los traductores con los que contactamos accedieron a participar en el estudio excepto uno, del que no obtuvimos respuesta.

Dada la diversidad geográfica de los infor- 
mantes, seis de las entrevistas se realizaron a distancia mediante el programa Skype, y el resto, en persona. Todas las entrevistas, que duraban una media de I hora y 23 minutos, fueron grabadas, previo consentimiento expreso de los informantes. Las cuestiones abordadas giraban en torno a dos grandes bloques:

I. Perfil y desarrollo profesional: se les preguntó por las vías de acceso a la traducción médica, el tipo de géneros y ámbitos que más traducen, las tareas que realizan, los requisitos que les exigen los empleadores, las dificultades que se encuentran, los recursos documentales que utilizan, los clientes para los que trabajan, entre otras.

2. Competencias: se les pidió que indicaran las competencias que consideraban necesarias para el ejercicio de la traducción médica.

Incluimos, además, un tercer bloque de preguntas destinadas a conocer la opinión de los informantes sobre la formación del traductor médico y la oferta formativa actual que, por limitaciones de espacio, no explicaremos en este artículo. No obstante, haremos alusión a los aspectos más relevantes a medida que expongamos los resultados de los otros dos bloques.

Las entrevistas fueron transcritas en su totalidad y se analizaron más de 9I.00o palabras. Para ello, se identificaron los fragmentos de las transcripciones que expresaban ideas relevantes para el estudio y se clasificaron según diferentes categorías temáticas. De este modo, surgieron categorías que en principio no se habían considerado, como su opinión sobre la evolución del mercado y la demanda, la cualificación del profesorado, el perfil formativo de otros compañeros, etc., que, de nuevo por motivos de espacio, no desarrollaremos en este trabajo.

\section{RESULTADOS DE LAS ENTREVISTAS}

A continuación, mostramos las principales respuestas de los informantes agrupadas según los bloques de la entrevista: perfil y desarrollo profesional, por un lado, y competencias destacadas, por otro.

\section{I. Perfil y desarrollo profesional \\ 4.I.I. Vias de acceso al campo de la traducción médica}

Los TFC accedieron a la traducción médica de manera totalmente casual, pues ninguno había previsto dedicarse a ella, y más habiendo estudiado medicina. Son varias las razones que motivan su acceso a esta actividad: la posibilidad de ganar un sobresueldo compaginando la traducción con la práctica clínica o con otras actividades no necesariamente vinculadas a la medicina (TFCI, TFC4 y TFC5), el desinterés por el ejercicio de la medicina $\left(\mathrm{TFC}_{2}\right)$ o la curiosidad por hacer cosas distintas $\left(\mathrm{TFC}_{3}\right)$. Además de las razones expuestas, todos los informantes manifestaban un interés especial por las lenguas.

Los TFL, por su parte, veían en la traducción médica una vía de especialización idónea para su desarrollo profesional y sus intereses personales, razón por la cual propiciaron el acceso a esta especialidad. La mayoría manifestaba también un claro interés por la medicina y las ciencias desde siempre (incluso un deseo frustrado por estudiar medicina, como señala TFL4), lo cual coincide con lo que les sucede a los TFC, pero a la inversa. Sólo TFL2 accedió al campo de la traducción médica gracias a estar especializado en la traducción de un género concreto: la patente.

\section{I.2. Adquisición de formación adicional}

La mayoría de informantes con formación científica no consideran imprescindible la formación reglada en traducción, pues todos ellos 
han ido resolviendo sus carencias lingüísticas a través de la formación autodidacta. Ninguno ha cursado estudios reglados de traducción aunque sí que han realizado a lo largo de su vida cursos de idiomas de diversa índole en escuelas oficiales, academias, etc. Algunos, incluso, como TFCI y $\mathrm{TFC}_{2}$, han estudiado en colegios bilingües.

En el caso de los TFL la situación es muy distinta, pues casi todos ellos manifiestan una necesidad evidente de adquirir conocimientos adicionales, en este caso temáticos, para realizar traducciones médicas: «yo lo que mayormente necesitaba era conocimientos médicos. Era lo que más me fallaba», señala TFLr. Aparte de la formación autodidacta, la mayoría considera que es esencial realizar un máster de traducción médica o cursos de medicina para no médicos para poder enfrentarse con mayor soltura a traducciones médicas complejas. No obstante, los que trabajan en plantilla ( $\mathrm{TFL}_{2}$ y $\mathrm{TFL}_{3}$ ) no consideran esta formación tan fundamental, pues suelen tener la posibilidad de delegar en traductores más especializados aquellos trabajos para los que se sienten menos preparados.

\section{I.3. Clientes para los que han trabajado}

Tanto los TFC como los TFL han trabajado para una amplia variedad de clientes del sector público y privado. La diversidad, como es obvio, es mayor entre los traductores autónomos que los de plantilla, que se mueven en terrenos mucho más acotados y recurrentes. Los clientes más mencionados del sector privado son: laboratorios farmacéuticos nacionales y extranjeros, centros de investigación, empresas de traducción, empresas de traducción especializadas en el ámbito médico-sanitario, CRO, editoriales, empresas relacionadas con la medicina (empresas de dispositivos y aparataje sanitario, de informática, etc.) y particulares (especialmente médicos e investigadores). Respecto al sector

público o institucional, destacan: los organismos internacionales (OMS, OPS, OMPI, Comisión Europea, etc.), los hospitales y los institutos de investigación.

A este respecto, existe una diferencia significativa entre los TFL y los TFC: a diferencia de los TFC, ninguno de los TFL había trabajado para editoriales (a excepción de los incipientes contactos que estaban empezando a establecer TFL5, TFL6 y TFL7 en el momento de la entrevista). Como curiosidad, cabe destacar el rechazo casi unánime de los traductores autónomos, especialmente los TFL, hacia las empresas de traducción, principalmente las españolas («las agencias te explotan vilmente», afirmaba TFL 7), aunque reconocen su utilidad para adquirir experiencia durante los primeros años de carrera profesional.

\section{I.4. Géneros textuales traducidos}

La gran mayoría de géneros textuales que traducen emana de la industria farmacéutica, lo cual corrobora la productividad de este sector. Estos géneros son de grados de especialización muy diversos: desde los más complejos, dirigidos a especialistas, como los protocolos de ensayos clínicos, hasta los más divulgativos, como las notas de prensa o los cuestionarios de calidad de vida. Los géneros que más traducen al español son:

- libros de medicina

- artículos de investigación

- expedientes de registro de medicamentos

- resúmenes de artículos científicos

- notas de prensa

- cuestionarios de calidad de vida

- textos publicitarios sobre productos farmacéuticos

- protocolos de ensayos clínicos

- información para pacientes 
- prospectos de medicamento

- documentos variados sobre salud pública.

Otros géneros que también se mencionan, aunque en menor medida, son: informes médicos, libros de anatomía, tratados y libros de texto médicos, consentimientos informados, estudios de mercado para empresas farmacéuticas, cartas, instrucciones de dispositivos médico-sanitarios, patentes, artículos divulgativos o fichas técnicas. Finalmente, se alude también a la traducción de géneros médico-jurídicos (Borja, 20I2), como reglamentos, decisiones, resoluciones, documentos de trabajo, contratos o poderes, los cuales se generan principalmente en la industria farmacéutica, los organismos internacionales y las instituciones europeas.

La diferencia principal entre los TFC y los TFL es que son los primeros los que suelen traducir libros y tratados, géneros prototípicos del sector editorial que, como veíamos en el apartado 4.I.3., parece restringido a los TFC. De hecho, los comentarios de los informantes evidencian que la formación médica es uno de los principales incentivos de las editoriales, a veces incluso más que la experiencia. Así lo señala TFC3:

[...] la editorial en general siempre se volcó en un perfil de traductor basado en el profesional técnico, es decir, médico, psicólogo, bioquímico o alguien que entendiera bien la materia de que se tratara, de manera que la titulación de médico la valoraron. La experiencia no, porque lo primero que les dije fue que no tenía experiencia $[. .$.$] Entonces estaban traducien-$ do un libro muy extenso de dermatología. Y al ser yo dermatólogo les interesó, porque lógicamente supusieron que yo podría traducir bien dermatología.

También existen diferencias entre los traductores de plantilla para organismos internacionales y los autónomos: los primeros traducen una menor variedad de géneros, muchos son de carácter jurídico-administrativo $\mathrm{y}$, en general, son menos especializados.

\section{I.5. Temas traducidos}

La mayoría de informantes, a excepción de TFL2, se dedica casi exclusivamente a la traducción médica. Los TFC traducen únicamente temas relacionados con la medicina o afines (farmacología, biología molecular, genética, etc.), mientras que los TFL suelen abarcar también otras especialidades de traducción (especialmente jurídica y técnica), sobre todo al inicio de su carrera profesional y hasta que se especializan en el ámbito médico-sanitario.

Respecto a los temas médicos que traducen, estos son muy variados: farmacología, salud pública, medicina interna, traumatología, ortopedia, etc. Los TFL consideran que temas como la cirugía o la descripción de instrumental quirúrgico son particularmente complejos y prefieren evitarlos. Los TFC también muestran cierto respeto por temas que no han estudiado o que son muy novedosos y que evolucionan muy rápido, como la biología molecular o la genética.

Por otra parte, los TFL destacan la importancia de contar con la revisión de expertos en la materia como solución óptima para asegurar la calidad de las traducciones, especialmente las de los temas más especializados. Así, TFL4 señala: «Paso a revisar cosas. Para mí es fundamental. [...] lo que tengo de biología molecular que no me queda nada claro, lo revisa X. [...] Aprendes muchísimo. Y mejoras un montón tu trabajo, porque siempre hay errores.» TFL5, TFL6 y TFL7, por su parte, se quejan de que los TFC no valoran el trabajo en tándem entre el especialista en el tema y el experto en traducción. A pesar de ello, existe consenso entre los informantes al afirmar que la situación ideal para solucionar este tipo de dificultades sería la colaboración 
entre traductores y expertos en la materia.

Finalmente, la particularidad que presentan los traductores de plantilla con respecto a los autónomos es que, como generalmente cuentan con el apoyo de un equipo de traductores externos de perfiles formativos diversos (farmacólogos, biólogos, médicos especialistas, etc.), pueden delegar en estos aquellos temas para los que no se sienten especialmente preparados.

\section{I.6. Combinaciones lingüisticas}

Todos los informantes traducen casi exclusivamente del inglés, a excepción de uno (TFL2), que, por su perfil particular, traduce mucho del chino. Este hecho parece confirmar que el inglés es el idioma por excelencia de la comunicación médica. El alemán también es un idioma de producción médica, aunque en un porcentaje mucho menor que el inglés (TFCI, TFC3 y TFC4 traducen esporádicamente del alemán). Respecto a la lengua meta, según los informantes existe mucha demanda de traducción al español, debido fundamentalmente al mercado hispanoamericano.

En cuanto a la traducción inversa, sólo dos informantes (TFCI y TFL4) traducen esporádicamente al inglés, principalmente artículos de investigación y resúmenes de artículos de investigación.

\subsubsection{Realización de otras tareas relacionadas con la traducción médica}

Además de la traducción, la revisión es una de las tareas que más realizan, ya que siete de los doce informantes revisan traducciones de forma habitual. TFC5 y TFLi aluden, además, a la realización de adaptaciones para pacientes de artículos médicos, una tarea que parece que cada vez va cobrando más peso gracias a la democratización del conocimiento y la tendencia a la educación del paciente (Muñoz Miquel, 20I2). Otras tareas mencionadas, aunque en menor medida, son la docencia (TFCI y TFL2), la redacción de textos médicos originales $\left(\mathrm{TFC}_{3}\right)$, la lexicografía $\left(\mathrm{TFC}_{7}\right)$ y la interpretación, que se realiza de manera muy esporádica (TFC4 y TFL6).

Finalmente, es destacable la diferencia que existe entre los traductores de plantilla y los autónomos, en tanto que los primeros realizan un abanico más amplio de tareas, como la creación de glosarios y guías de estilo, la edición, la gestión de proyectos o la selección de traductores y colaboradores externos.

\section{I.8. Requisitos exigidos por parte de los empleadores}

Al igual que sucede con el ejercicio de la traducción en general (Pym et al., 2012: 20 y ss.), es posible acceder al mercado de la traducción médica desde cualquier titulación. Por lo general, los candidatos muestran su valía a través de una prueba de traducción.

Existen, no obstante, determinados requisitos de acceso que difieren según el tipo de empleador de que se trate. Para acceder a organismos internacionales es necesario superar una oposición, que implica la realización de pruebas diversas: exámenes de traducción y de conocimientos generales, entrevistas, acreditación de un número determinado de años de experiencia, etc. Estos criterios se tornan algo más laxos cuando se trata de seleccionar a colaboradores externos para trabajar en dichos organismos, para lo que se procura que el perfil de estos sea lo más variado posible. En el caso de las editoriales, como ya hemos visto (apartados 4.I.3. y 4.I.4.) parece existir una clara preferencia por los TFC, ya que es menos frecuente que contraten a traductores con formación lingüística, a no ser que tengan una dilatada experiencia en el campo de la traducción médica. Parece, pues, que la formación médica o científica se valora 
sustancialmente y, en muchos casos, se prefiere: «[...] yo creo que tener la formación formal en medicina se valora mucho», afirma $\mathrm{TFC}_{2} ;$ «[...] lo que pesó bastante en ese entonces fue el título de biólogo molecular», señala TFC5.

\section{I.9. Principales dificultades de la traducción médica}

La comprensión de conceptos especializados se configura como el principal problema para diez informantes. Los dos que no lo mencionan (TFL2 y TFL3) trabajan para organismos internacionales $\mathrm{y}$, como hemos comentado, pueden delegar en colaboradores externos los textos más complejos, lo cual podría explicar que la comprensión de conceptos especializados no sea su principal dificultad. En este sentido, TFCI, TFC2 y TFLI puntualizan que la tarea de documentación difícilmente puede suplir la falta de conocimientos médicos básicos.

Otra de las dificultades que nombran diez de los doce informantes tiene que ver con la equivalencia terminológica. El principal problema radica en saber discernir qué término, de entre varias opciones, se debe escoger según el contexto. Ser capaz de mimetizar la fraseología y la manera de expresarse de los especialistas a los que va dirigido el texto también es otra de las dificultades más mencionadas. Y, en este sentido, llegar a un justo equilibrio entre la manera de expresarse de los especialistas y la norma lingüística es también difícil, pero esencial. TFC3 afirma lo siguiente: «Para mí no vale mucho un texto que desde el punto de vista de la lengua española, en general, es correcto, pero que está lleno de palabras que al especialista en medicina le suenen raras porque no las usa».

La obtención de información fiable sobre temas muy novedosos y que están en constante evolución es también otra de las dificultades más notables, ya que, según indican, la infor- mación publicada en español suele ser escasa. La traducción de neologismos es un problema aparejado al anterior que también suele resultar muy complejo de resolver. Finalmente, la mala redacción de los originales y la ambigüedad del inglés médico es otro de los problemas más recurrentes, sobre todo entre los TFL.

\section{I.Io. Recursos de consulta utilizados}

La consulta a colegas y a expertos en la materia, especialmente a través de foros especializados o listas de debate, es un recurso fundamental para los traductores entrevistados, independientemente de la formación de base que tengan. Diez de los doce informantes consultan regularmente el foro terminológico de Tremédi$\mathrm{ca}^{9} \mathrm{y}$ el foro de Medtrad, ${ }^{\text {ro }}$ ambos especializados en traducción y redacción médico-sanitarias, para resolver dudas conceptuales y terminológicas. Como señala TFL4: «Medtrad y Tremédica son básicos para cualquier traductor que se meta en este ámbito».

Los diccionarios son también una herramienta esencial. Ocho informantes mencionan explícitamente el Diccionario crítico de dudas inglés-español de medicina, de F. A. Navarro, lo que pone de manifiesto su valor entre los que ejercen la traducción médica. También se mencionan con frecuencia el Diccionario de Términos Médicos de la Real Academia Nacional de Medicina y otros diccionarios monolingües, como el Stedman's Medical Dictionary o el Dorland's Illustrated Medical Dictionary. Otros recursos ampliamente utilizados son las bases de datos (Pubmed, MEdes, IATE...), los portales médicos (MD Consult, Medscape...), los textos

9 Asociación Internacional de Traductores y Redactores de Medicina y Ciencias Afines (http://www.tremedica. org/).

ro Foro de traductores profesionales de biomedicina (http://www.rediris.es/list/info/medtrad.html). 
paralelos, los manuales y tratados médicos, los glosarios de elaboración propia, así como documentación diversa publicada por organismos oficiales (plantillas de la Agencia Europea del Medicamento, guías de estilo, nomenclaturas, clasificaciones, etc.). Cabe destacar que únicamente son los informantes que trabajan en plantilla ( $\left.\mathrm{TFC}_{4}, \mathrm{TFC}_{5}, \mathrm{TFL}_{3}\right)$ los que mencionan las memorias y los programas de asistencia a la traducción (por ej., dtSearch) como una herramienta básica en su trabajo. Esto puede deberse a que el trabajo en estos organismos está bastante automatizado y a que los géneros que se traducen suelen ser muy recurrentes.

\section{I.II. Pertenencia asociaciones profesionales}

Diez de los informantes pertenecen a Tremédica y, según indican, pertenecer a una asociación permite establecer contactos con colegas de profesión y con expertos en la materia, estar al día de las novedades que acontecen en el ámbito, consultar dudas, e intercambiar ideas, inquietudes o problemas. TFC2 destaca, además, que las asociaciones y los foros de traducción fueron su escuela durante sus inicios, ya que, al haber estudiado medicina, desconocía por completo cómo funcionaba el mundo de la traducción. TFL4, TFL5, TFL6 y TFL7, por su parte, aluden a la tarea pedagógica de las asociaciones de cara, sobre todo, a orientar a los que empiezan, y opinan que, en este sentido, suplen muchas de las carencias de la formación universitaria.

\section{I.I2. Papel de la formación continuada}

Nueve informantes asisten con cierta regularidad a cursos o jornadas relacionados con la medicina o la traducción médica, como una forma de adquirir formación complementaria o, sencillamente, de mantenerse al día. En este sentido, se observa cierto malestar, sobre todo entre los TFL, respecto a la escasa oferta de

cursos específicos en traducción médica y la excesiva proliferación de posgrados generalistas: «los estudios universitarios tienen tendencia a perderse en generalidades y no profundizan», señala TFL7. TFL4, TFL5, TFL6 y TFL7 apuntan que asistir a congresos o cursos de medicina, aunque no estén orientados a traductores, es una buena alternativa para «empaparse», como dice TFL4, de terminología y de conocimientos temáticos. Además de todo esto, la formación autodidacta mediante la lectura de libros y tratados sobre el tema en cuestión es un recurso fundamental.

\subsection{Competencias destacadas}

Una vez vistas las características socioprofesionales de los traductores entrevistados, en este apartado recogemos su opinión sobre las competencias que consideran necesarias para ejercer la traducción médica. Estas competencias las hemos agrupado en cinco categorías, basándonos parcialmente en la denominación que reciben estas en propuestas como las de Kelly $(2002,2005),{ }^{\text {II }}$ PACTE $(2003)^{\mathrm{I2}}$ o Hurtado (2007). ${ }^{\mathrm{I}}$ Así, distinguiremos entre aspectos temáticos y terminológicos; aspectos documentales e instrumentales; aspectos socioprofesionales e interpersonales; aspectos actitudinales, y aspectos lingüísticos y textuales.

\subsection{Aspectos temáticos y terminológicos}

Diez de los informantes coinciden en señalar que es fundamental tener una base de conocimientos médicos: «alguien que se dedica a la traducción médica tiene que tener conoci-

II Comunicativa y textual, cultural, temática, instrumental profesional, psicofisiológica, interpersonal y estratégica.

${ }_{12}$ Bilingüe y extralingüística, de conocimientos de traducción, instrumental y estratégica.

${ }_{3}$ Estratégica y metodológica, contrastiva, extralingüística, profesional, instrumental y textual. 
miento técnico de la materia y conocimiento conceptual lo suficientemente profundo como para manejar bien el tema», afirma TFC3. Los traductores afirman que estos conocimientos de base facilitan la comprensión y la reexpresión de conocimientos especializados; ayudan a detectar posibles errores del original y a resolver problemas (como determinar cuándo un adjetivo afecta a todos los sustantivos que le siguen o sólo a uno); y agilizan notablemente el proceso de documentación. TFLi afirma: «si tuviese hechos dos o tres años de medicina, me ayudaría un montón»; por su parte, TFC2 señala que: «Si ya las personas con formación formal [en medicina] se pasan la vida buscando desesperadas [...], si no tienes preparación formal, el tiempo que vas a tener que dedicar a buscar no te compensa».

La adquisición de estos conocimientos se hace imprescindible para la traducción de géneros muy especializados, y menos necesaria en el caso de la divulgación:

Si es un texto especializado, yo creo que no hay duda: es muy difícil que un traductor lo pueda traducir sin conocimientos de medicina. (TFCI).

Yo creo que sí puedes hacer traducción biosanitaria divulgativa teniendo una formación no formal en medicina o en farmacia. Se puede hacer, a base, por supuesto, de aprender $\mathrm{y}$ de estudiar. (TFC2).

Debido a la importancia que se otorga a los conocimientos temáticos, algunos de los informantes, especialmente los TFC, consideran que especializarse en traducción médica es mucho más sencillo si se parte de una formación científica o médica: «el camino que tienes que recorrer [siendo traductor de formación] es más largo», señala TFL4. TFC2 sugiere, incluso, que sean las facultades de medicina las que oferten posgrados de traducción médica.

La adquisición de conocimientos sobre terminología y fraseología médicas también es, a juicio de los informantes, una competencia fundamental para poder mimetizar la forma de expresarse del colectivo al que vaya dirigido la traducción. Como señala TFC2:

[...] todo lo que se trabaje la terminología médica es poco. [...] Un traductor que sea capaz de utilizar con una gran precisión la terminología especializada en un texto dirigido a expertos conseguirá que ese texto parezca escrito por un experto. Y eso se ve enseguida, sobre todo en algunas especialidades [...]. Enseguida se ve si el texto lo ha escrito alguien que no está muy ducho en la materia, aunque sea muy correcto.

Seis de los informantes afirman que adquirir esa «capacidad de mimetización» es, incluso, más difícil que adquirir conocimientos médicos puros.

\subsubsection{Aspectos documentales e instrumentales}

Conocer las principales fuentes de documentación médico-sanitaria (como las citadas en el apartado de 4.I.ro.) y saber a qué recurso acudir según el tipo de dificultad es, dentro de esta categoría, la competencia que destacan todos los informantes. Por otra parte, ante la gran cantidad de información que se puede obtener de Internet, consideran imprescindible saber identificar las fuentes que realmente son fiables y de calidad, saber aplicar estrategias de búsqueda avanzada o, como señala TFC2, conocer «los trucos para eludir el hecho de que muchos documentos originales no sean accesibles más que pagando, que es un problema importante que tenemos con frecuencia».

Cabe destacar que ninguno de los informantes menciona competencias relacionadas con el dominio de herramientas o programas de asis- 
tencia a la traducción o a la gestión terminológica, algo que tal vez sí podría ser más relevante en otros ámbitos, como el audiovisual, en el que el dominio de determinados programas informáticos resulta esencial (Cerezo, 20I2). El único comentario al respecto lo realiza el informante TFL6, que considera peligroso el uso acrítico de los programas de traducción asistida y el hecho de que las nuevas generaciones de traductores estén adaptando su manera de traducir a este tipo de herramientas.

\subsubsection{Aspectos socioprofesionales e interpersonales}

Tener un conocimiento profundo del mercado de la traducción médica tanto a nivel nacional como internacional es uno de los aspectos más valorados por los informantes. Diez de ellos señalan que se han de conocer las características de los sectores existentes (industria farmacéutica, editorial, salud pública, investigación, etc.) y los aspectos más valorados en cada uno de ellos, pues ello determina en gran medida:

- la forma de trabajar y de enfocar las traducciones y el tipo de conocimientos que se necesitan;

- la manera de desenvolverse con los clientes y con otros profesionales que puedan estar implicados en el proceso de traducción de dicho sector, $y$

- la posibilidad de encontrar nuevos clientes o nichos de mercado.

\section{Así, TFC2 afirma que:}

[...] no tiene nada que ver trabajar para un organismo internacional que para laboratorios, que para una agencia especializada, que para una agencia general, que para un cliente particular [...]. Son mundos completamente distintos y ese aspecto del mundo real yo no sé si se les enseña a los traductores en su formación, pero me parece muy importante.

Asimismo, TFL4 señala que «es interesante conocer muchos sectores, porque cada uno tiene sus especificidades, y uno ya puede saber dónde encaja más», y subraya algunos de los rasgos característicos de la industria farmacéutica y de los organismos internacionales que pueden influir en la manera de trabajar del traductor:

Bueno, es que no te pases ni 15 minutos, si quieres mantener un cliente tan exigente, competitivo y serio como es la industria farmacéutica. [...] la calidad desde el punto de vista terminológico y estilístico es secundaria a la puntualidad y a la funcionalidad del texto.

Los organismos internacionales [...] mueven mucho trabajo. $\mathrm{Y}$ es un tipo de trabajo totalmente distinto [...] Ellos tienen un estilo muy rígido, muy calcado, porque los textos se tienen que parecer mucho en los dos idiomas. Entonces, donde hay una sigla, tiene que ir una sigla.

Saber adecuarse a los requisitos del encargo y del cliente, y, muy especialmente, ser capaz de cumplir con los plazos de entrega, es una competencia muy valorada por los informantes. Saber tratar de manera profesional tanto con clientes como con colegas y expertos en la materia también es una habilidad destacada por la mayoría. Asimismo, se hace especial hincapié en la importancia de saber cómo plantear dudas en foros de traductores y de expertos, y perder el miedo y la vergüenza a preguntar. Por otra parte, se destaca también la capacidad para justificar y argumentar las opciones de traducción y las decisiones tomadas, y para resolver los posibles conflictos que puedan surgir con el cliente. La capacidad de trabajo en equipo es una competencia que destacan especialmente los traductores de plantilla. 
Otras cuestiones a las que aluden los informantes son: saber qué tarifas aplicar según el tipo de cliente o encargo, saber cómo gestionar la cartera de clientes, y tener conocimientos básicos de fiscalidad y de deontología profesional.

\subsubsection{Aspectos lingüisticos y textuales}

El dominio de las lenguas de trabajo y, muy especialmente, de la lengua materna, es otra de las competencias que destacan nueve de los doce informantes. Entendemos que quienes no aluden explícitamente a ella ( $\mathrm{TFC}_{4}, \mathrm{TFC}_{5}$ y TFLI) la dan, sencillamente, por supuesta. El «respeto» y la «sensibilidad» por la lengua materna, como señalan TFCI, TFC2, TFL5, TFL6 y TFL7, se concreta en una serie de habilidades: la capacidad para realizar lecturas críticas y análisis textuales que permitan determinar las características del encargo; el dominio de los diferentes registros de la lengua, especialmente el científico, y la capacidad para redactar de manera comprensible y fluida.

El conocimiento de las características y convenciones de los géneros médico-sanitarios más demandados es otra de las competencias mencionadas por la mayoría de los traductores. Esta, además, ayuda al traductor a ser consciente del abanico tan amplio de posibilidades de trabajo que ofrece la traducción médica:

[...] mostrarle a un traductor biosanitario la enorme variedad de géneros textuales le ayuda también a darse cuenta de la amplitud del campo y de las posibilidades [...]. Puede hacer todo tipo de cosas, desde cuestiones farmacéuticas e incluso preclínicas [...] hasta aspectos de lo más básicos referidos al paciente. $\left(\mathrm{TFC}_{2}\right)$

En general, los comentarios de los informantes evidencian la pertinencia del concepto de género para la práctica de la traducción profe- sional, aunque no todos ellos hagan referencia explícita al término género en sí.

4.2.5. Aspectos actitudinales y psicofisiológicos

Finalmente, las actitudes y los componentes psicofisiológicos que destacan los informantes son: el sentido común, aspecto destacado de manera unánime; la curiosidad y el deseo de estar al día de todas las novedades que acontecen en el ámbito; el afán por la calidad y la perfección; la capacidad de organización y de automatización de las tareas más habituales; la capacidad de reflexión, y la actitud crítica.

\section{INTERPRETACIÓN Y CONCLUSIONES}

Como hemos visto, el acceso a los profesionales nos ha permitido obtener información amplia y variada sobre el ejercicio de la traducción médica, en general, y sobre el perfil y las competencias del traductor médico, en particular. A pesar de que, por la naturaleza cualitativa del estudio, no podemos llegar a conclusiones generalizables, estos resultados nos permiten apuntar tendencias acerca de los aspectos estudiados.

En primer lugar, se ha observado que el perfil formativo influye en el desarrollo profesional. Algunas diferencias significativas entre los TFC y los TFL son las siguientes:

- Los TFC acceden de manera fortuita a la traducción médica, actividad que compaginan con otro trabajo principal. Esto pone de manifiesto que, a pesar de que la traducción médica no es una salida natural de los médicos o científicos, estos acaban ocupando este campo gracias al valor que el mercado y los propios profesionales otorgan a la formación médico-científica. Esta parece, pues, su ventaja competitiva. En el caso de los TFL, la traducción médica es una vía de especialización 
propia de la formación en traducción por la que se suelen inclinar quienes tienen un interés especial por los temas médico-sanitarios. Hay, por tanto, un componente vocacional fundamental.

- Ambos perfiles formativos necesitan adquirir formación adicional para suplir sus carencias (lingüísticas y traductológicas, en el caso de los TFC; temáticas y terminológicas, en el caso de los TFL). La formación autodidacta es lo más socorrido en ambos casos, aunque son los TFL los que manifiestan la conveniencia de adquirir la formación médica básica que les falta a través de la formación reglada (cursos de medicina, posgrados de traducción médica, etc.). Se evidencia, por tanto, que la carrera de traducción no sería suficiente para afrontar traducciones médicas especializadas. Para los TFC, la formación autodidacta en lenguas parece ser suficiente, pues lo que prima es la formación médica. Esta primacía podría deberse a la posición privilegiada que ocupa la medicina como profesión clásica por excelencia (Freidson, I988).

- El hecho de que la traducción no sea una salida profesional propia de los TFC explicaría por qué estos sólo traducen medicina, mientras que los TFL abarcan también otras especialidades de traducción. Con todo, la traducción médica parece ofrecer suficientes posibilidades de trabajo como para que los TFL también puedan ganarse la vida traduciendo exclusivamente esta especialidad.

- El sector editorial parece estar vetado a los TFL, no sólo porque las editoriales prefieren a los expertos en el ámbito temático, sino porque ellos mismos sienten cierto respeto hacia la traducción de géneros propios del sector, dada la inseguridad que les produce la falta de conocimientos especializados. Como señalaba TFLr: «nunca me lo han ofrecido, pero yo un manual de medicina no me atrevo a traducirlo».

Los resultados muestran también algunas diferencias entre los traductores autónomos y los de plantilla:

- Los organismos que tienen traductores de plantilla procuran cubrir con colaboradores externos un amplio abanico de especialidades médicas, razón que justificaría por qué los primeros no necesitan tantos conocimientos médicos especializados como los autónomos.

- Los traductores autónomos, sin embargo, no realizan tareas tan variadas como los de plantilla, que, con frecuencia: realizan revisiones y controles de calidad de las traducciones de los externos, crean glosarios y libros de estilo, seleccionan a los colaboradores, y editan textos. Como vemos, la figura del traductor médico de plantilla tiene muchos puntos en común con la del gestor de proyectos.

Aparte de todas estas diferencias, los traductores médicos, independientemente de su perfil formativo o profesional, tienen ciertos rasgos característicos:

- Se encargan de un abanico de clientes y de géneros textuales muy amplio, principalmente del sector farmacéutico, cuya demanda pueden satisfacer traductores con diferentes niveles de especialización. No obstante, cuanta mejor preparación temática se tenga, más posibilidades tendrán de responder a una mayor variedad de encargos.

- Hacen frente a una serie de dificultades, entre las que destacan la comprensión de conocimientos temáticos especializados y la utilización adecuada de la terminología y la fraseología. Para resolverlas, otorgan suma 
importancia al uso de recursos de consulta documental pero, especialmente, humana. Los colegas y expertos en la materia se convierten, por tanto, en los mejores aliados de los traductores médicos.

- Valoran notablemente la pertenencia a asociaciones y la asistencia regular a jornadas y cursos de formación continuada para aumentar su formación y mantenerse al día de las novedades del ámbito.

- Comienzan a encargarse de tareas, como la adaptación de investigaciones originales para pacientes y público general, que, a priori, no se considerarían traducciones en el sentido más tradicional de la palabra, pero que responden a las necesidades de la cultura sanitaria actual, tendente a la educación al paciente (Muñoz Miquel, 20I2: 204)

Respecto a las competencias, los informantes, independientemente de su perfil formativo, coinciden en hacer primar las siguientes:

- tener conocimientos médicos básicos, lo cual está vinculado a un profundo conocimiento de la terminología y la fraseología médicas y del tema que se traduce;

- conocer las principales fuentes documentales del ámbito médico, saber identificar los recursos fiables y dominar las estrategias de documentación avanzadas;

- tener un profundo conocimiento del mercado de la traducción médica;

- dominar las lenguas de trabajo, especialmente la materna;

- saber socializar con expertos y colegas del ramo;

- ser capaz de ajustarse a las características del encargo y a los plazos de entrega;

- tener sentido común y una excelente capacidad de organización.
Como podemos ver, estos datos nos permiten tener una visión más objetiva del ejercicio profesional de la traducción médica. Aunque somos conscientes de que provienen de una muestra reducida de informantes y de que no pueden extrapolarse al conjunto de la población, consideramos que pueden servir para tomar decisiones más informadas sobre las competencias que deberían adquirir los estudiantes en un programa de especialización en traducción médica. Así, según los resultados obtenidos, estos programas deberían incluir, entre otros: contenidos médicos básicos, prácticas de traducción de los géneros que tienen más peso en el mercado (como los de la industria farmacéutica), nociones de terminología, aspectos sobre el mercado de la traducción médica o tareas en las que los estudiantes deban colaborar con expertos en la materia. En trabajos futuros llevaremos a cabo un estudio cuantitativo con encuestas dirigidas a una muestra de población más amplia para confirmar todas estas conclusiones.

RECIBIDO EN ABRIL DE 2013

ACEPTADO EN SEPTIEMBRE DE 2013 VERSIÓN FINAL DE DICIEMBRE DE 2013

\section{REFERENCIAS BIBLIOGRÁFICAS}

Albarrán Martín, R. (20I2). Traducción y medicina: aspectos epistemológicos e interdisciplinares para la formación de traductores especializados en ciencias de la salud. Tesis doctoral, Salamanca: Universidad de Salamanca.

Alarcón Navío, E. (I998). «Variedad y especificidad de la traducción médica». En Félix Fernández, L. y E. Ortega Arjonilla (coords.): II Estudios sobre Traducción e Interpretación, Málaga: Universidad de Málaga, pp. Io25-1034.

Bloom, B. (1985). Developing Talent in Young People, New York: Ballantine.

Borja Albi, A. (20I2). «Aproximación traductológica a los textos médico-jurídicos».Panace@, 13/36, pp. I67-I75. 
Calvo Encinas, E. (2010). Análisis curricular de los estudios de Traducción e Interpretación en España. Perspectiva del estudiantado. Tesis doctoral, Granada: Universidad de Granada.

180 Cerezo Merchán, B. (2012). La didáctica de la traducción audiovisual en España: Un estudio de caso empírico-descriptivo. Tesis doctoral, Castellón de la Plana: Universitat Jaume I.

Creswell, J. W. (2007). Qualitative inquiry and research design: choosing among five approaches, Thousand Oaks: Sage Publications.

Fischbach, H. (ed.) (1998). Translation and Medicine, Amsterdam: John Benjamins.

Franco Aixelá, J. (20I0). «Una revisión de la bibliografía sobre traducción e interpretación médica recogida en BITRA (Bibliografía de Interpretación y Traducción)».Panace@, II/32, pp. I5I-I6o.

Freidson, E. (I988). Profession of Medicine, Chicago: The University of Chicago Press.

Gamero Pérez, S. (200I). La traducción de textos técnicos, Barcelona: Ariel.

González, J.y R. Wagenaar (2003). Tuning educational Structures in Europe. Final report. Pilot Project. Phase one, Bilbao: Universidad de Deusto.

Hurtado Albir, A. (2007). «Competence-based Curriculum Design for Training Translators». The Interpreter and Translator Trainer (ITT), I/2, pp. I63-I95.

Kelly, D. (2002). «Un modelo de competencia traductora: bases para el diseño curricular». Puentes, I, pp. 9-20.

- (2005). A Handbook for Translator Trainers, Translation Practices Explained Series, Manchester: St. Jerome Publishing.

Lee-Jahnke, H. (1998). «Training in Medical Translation with Emphasis on German». En Fischbach, H. (ed.) (I998: 8I-9I).

- (200I). «L'enseignement de la traduction médicale: un double défi?». Meta, 46/I, pp.145-153.

Lozano, M. F. (2009). «La transformación del papel del traductor en la Organización Panamericana de la Salud». En Unión Latina y Red Mundial para la Diversidad Lingüística Maaya (eds.): Actas del Congreso Mundial de Traducción Especializada "Lenguas y diálogo intercultural en un mundo en globalización», París: Unión Latina, pp. 87-91.

Mayoral Asensio, R. (200I). Aspectos epistemológicos de la traducción, Castellón de la Plana: Universitat Jaume I.
Mayoral Asensio, R. y O. Diaz Fouces (20II). La traducción especializada y las especialidades de la traducción, Castellón de la Plana: Universitat Jaume I.

Montalt, V. (2007). «La enseñanza virtual de la traducción médica en el espacio europeo de educación superior».Panace@, 8/26, pp. 213-219.

- (2009). «La consulta documental y humana aplicada a la traducción médica: Reflexiones en torno a la práctica profesional y a la pedagogía». En Pinto Molina, M., Sales Salvador, D., Planelles, E. y N. Bas (eds.): Documentación aplicada y Espacio Europeo de Educación Superior, Madrid: Arco Libros, pp. I7I-I86.

Montalt, V. y M. González Davies (2007). Medical Translation Step by Step: learning by drafting, Manchester: St. Jerome Publishing.

Muñoz Miquel, A. (2009). «El perfil del traductor médico: diseño de un estudio de corte socioprofesional».Panace@, 10/30, pp. 157-167.

- (20I2). «From the original article to the summary for patients: reformulation procedures in intralingual translation», Linguistica Antverpiensia, II, pp.187-206.

Muñoz, A., Montalt, V. e I. García Izquierdo (20I2). «La investigación socio-profesional y la competencia traductora aplicadas a la pedagogía de la traducción médica». En Cruces Colado, S., Del Pozo Triviño, M., Luna Alonso, A. y A. Álvarez Lugrís (eds.): Traducir en la frontera, Granada: Atrio, pp. IO3-I20.

Muñoz Raya, E. (coord.) (2004). Libro Blanco. Titulo de Grado en Traducción e Interpretación. Agencia Nacional de Evaluación de la Calidad y Acreditación [en línea]. Granada: Universidad de Granada < http://www.aneca.es/var/media/I50288/libroblanco_traduc_def.pdf $>$ [Consulta: 30/II/20I3].

Navarro, F. A. (2002). "Traductores profesionales y profesionales traductores en los albores de una nueva era». En Actas del I Congreso Internacional: El español, lengua de traducción [en línea]. Almagro: Comisión Europea y Agencia EFE < http:// cvc.cervantes.es/lengua/esletra/pdf/or/o23_navarro.pdf> [Consulta: $\left.\mathrm{O}_{2} / \mathrm{O}_{4} / 2 \mathrm{OI} 3\right]$.

Navarro, F. A. y F. Hernández (I997). «Anatomía de la traducción médica». En Félix Fernández, L. y E. Ortega Arjonilla (coords.): Lecciones de teoria y práctica de la traducción, Málaga: Universidad de Málaga, pp. I37-I62. 
O’Neill, M. (1998). «Who Makes a Better Medical Translation: The Medically Knowledgeable Linguist or the Linguistically Knowledgeable Medical Professional? A Physician's Perspective». En Fischbach, H. (ed.) (1998: 69-80).

Orf, D. (2005): Estudio de situación del mercado español de servicios profesionales de traducción, Madrid: Agrupación de Centros Especializados en Traducción.

PACTE (2003). «Building a Translation Competence Model». En Alves, F. (ed.): Triangulating Translation: Perspectives in process oriented research, Amsterdam: John Benjamins, pp. 43-66.
Pilegaard, M. (2000). «Medical LSP: An introduction to the thematic section». Hermes, Journal of Linguistics, 25, pp. 7-9.

Pym, A., Grin, F., Sfreddo, C., y A. L. J. Chan (2012). The status of the translation profession in the European Union (DGT/20II/TST). Final Report, Luxembourg: European Commission.

Rouleau, M. (2012). La traduction médicale: Une approche méthodique, Montreal: Linguatech.

Yániz Álvarez de Eulate, C. y L. Villardón Gallego (2006). Planificar desde competencias para promover el aprendizaje, Bilbao: Mensajero. 